\title{
Synthesis of Hydrated Ternary Lanthanide-Containing Chlorides Exhibiting X-ray Scintillation and Luminescence
}

\author{
Gyanendra B. Ayer ${ }^{1}$, Mark D. Smith ${ }^{1}$, Luiz G. Jacobsohn ${ }^{2}$, Gregory Morrison ${ }^{1}$, Hunter \\ B. Tisdale ${ }^{1}$, Logan S. Breton ${ }^{1}$, Weiguo Zhang ${ }^{3}$, P. Shiv Halasyamani ${ }^{3}$, and Hans-Conrad zur \\ Loye*1 \\ ${ }^{1}$ Department of Chemistry and Biochemistry, University of South Carolina, 631 Sumter \\ Street, Columbia, SC, United States \\ *e-mail: zurloye@mailbox.sc.edu \\ ${ }^{2}$ Department of Materials Science and Engineering, Clemson University, Clemson, SC, USA \\ ${ }^{3}$ Department of Chemistry, University of Houston, Houston, TX, United States
}




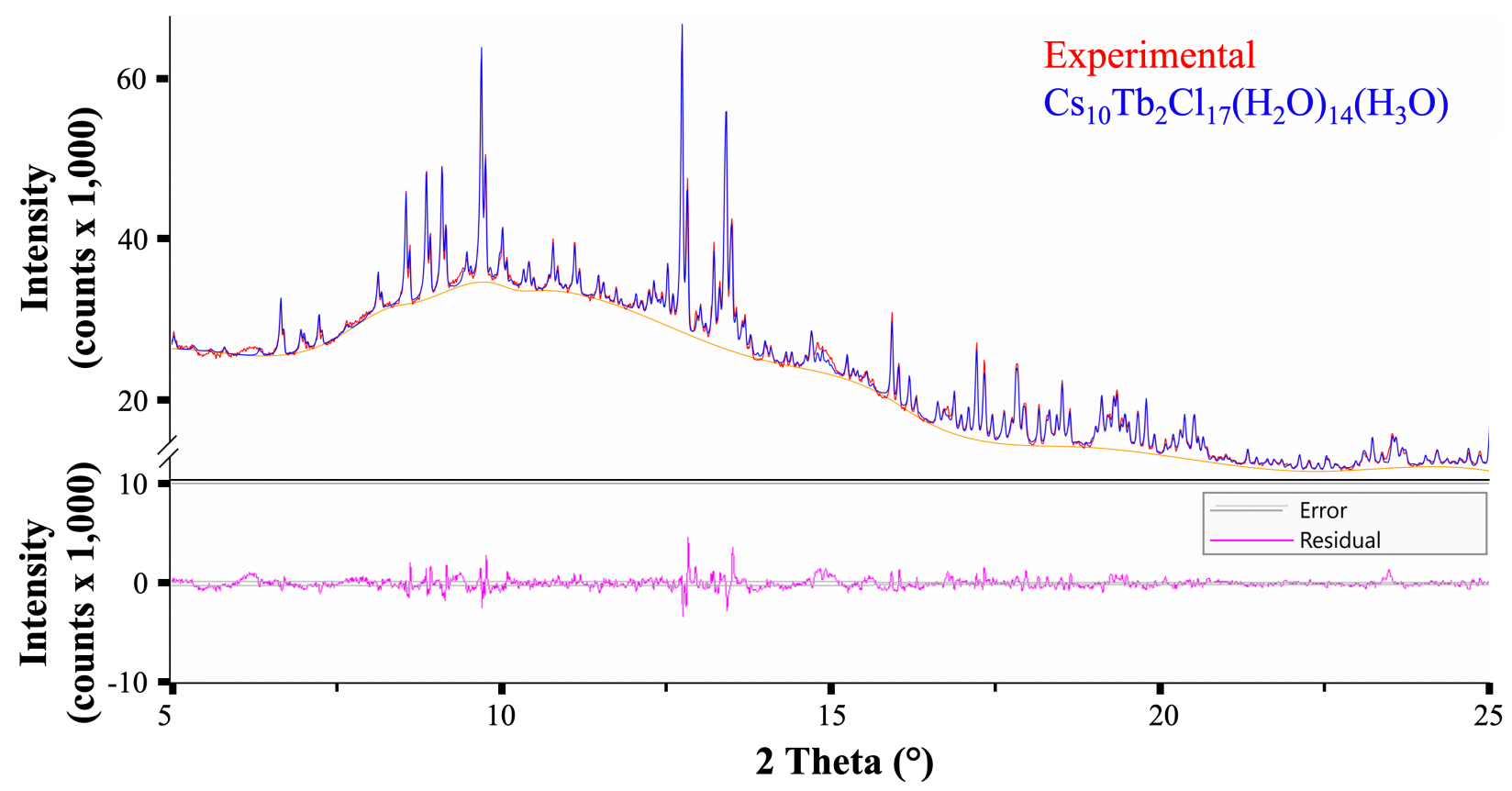

\begin{tabular}{|c|c|c|c|}
\hline \multicolumn{4}{|c|}{$\mathrm{Cs}_{10} \mathrm{~Tb}_{2} \mathrm{Cl}_{17}\left(\mathrm{H}_{2} \mathrm{O}\right)_{14}\left(\mathrm{H}_{3} \mathrm{O}\right)$ Lattice Parameters } \\
\hline & $\begin{array}{c}\text { SXRD Structure } \\
\text { Solution } \\
(\mathbf{1 0 0 K})\end{array}$ & $\begin{array}{c}\text { SXRD Unit Cells } \\
\text { Checks } \\
(\mathbf{2 9 8 K})\end{array}$ & $\begin{array}{c}\text { PXRD Rietveld/d-I } \\
\text { pattern refinement } \\
(\mathbf{2 9 8 K})\end{array}$ \\
\hline $\mathrm{a}(\AA)$ & $18.1569(5)$ & $18.33(1)$ & $18.3250(13)$ \\
\hline $\mathrm{b}(\AA)$ & $17.8077(5)$ & $17.83(3)$ & $17.8531(13)$ \\
\hline $\mathrm{c}(\AA)$ & $16.5082(5)$ & $16.64(1)$ & $16.6446(12)$ \\
\hline Volume $\left(\AA^{3}\right)$ & 5337.640 & 5439 & 5445.425 \\
\hline
\end{tabular}

Figure S1. PXRD data for $\mathrm{Cs}_{10} \mathrm{~Tb}_{2} \mathrm{Cl}_{17}\left(\mathrm{H}_{2} \mathrm{O}\right)_{14}\left(\mathrm{H}_{3} \mathrm{O}\right)$ showing the diffraction pattern and calculated pattern using the Rietveld/d-I pattern method (top) and the residual intensity (middle) and a table of lattice parameters (bottom). Note that this data was collected using Mo K $\alpha$ X-rays $(\boldsymbol{\lambda}=$ $0.70932 \AA$ ). Therefore, diffraction angles cannot be directly compared to tradition $\mathrm{Cu} \mathrm{K} \alpha \mathrm{PXRD}$ patterns without conversion. Furthermore, due to the high-resolution nature of the instrument, $\mathrm{K} \alpha 1 / \mathrm{K} \alpha 2$ splitting is observed even for low angle diffraction peaks. 


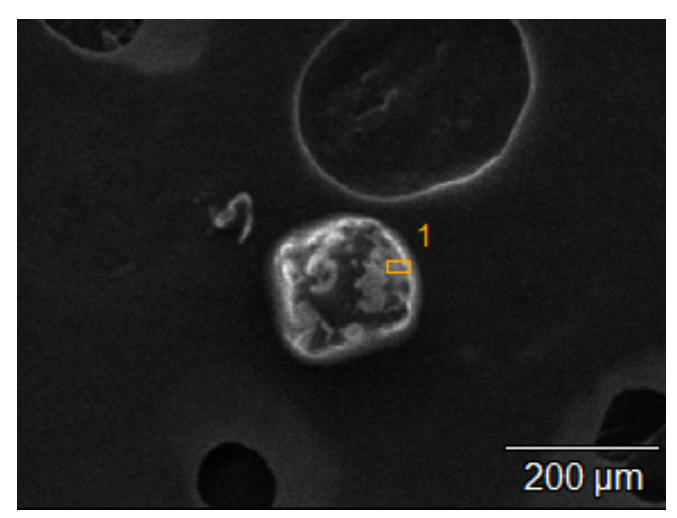

(a)

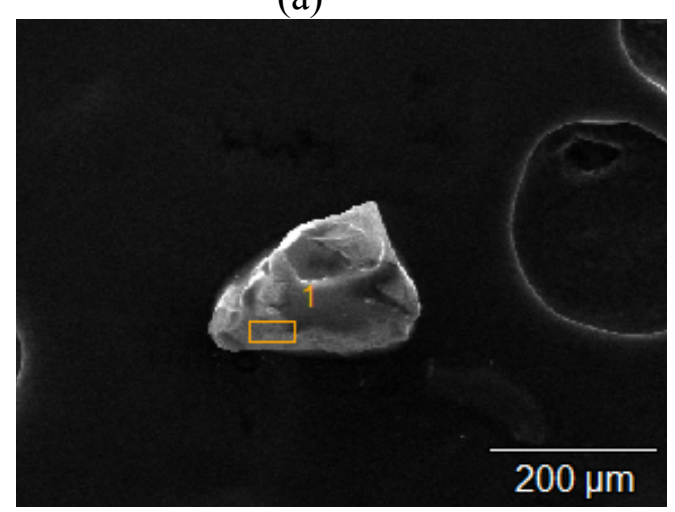

(c)

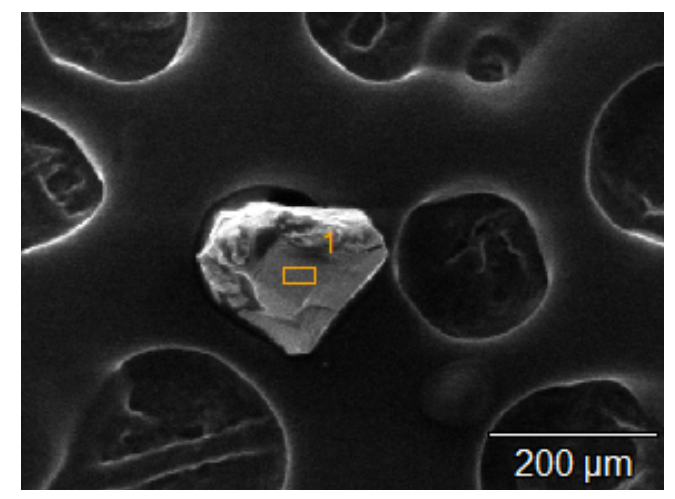

(e)

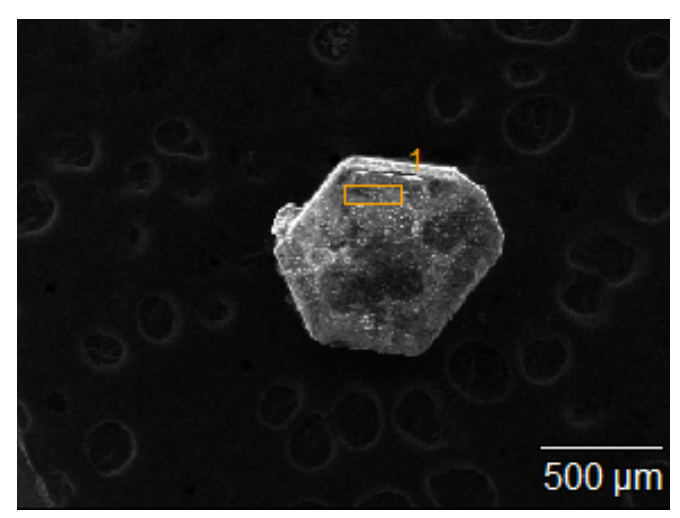

(b)

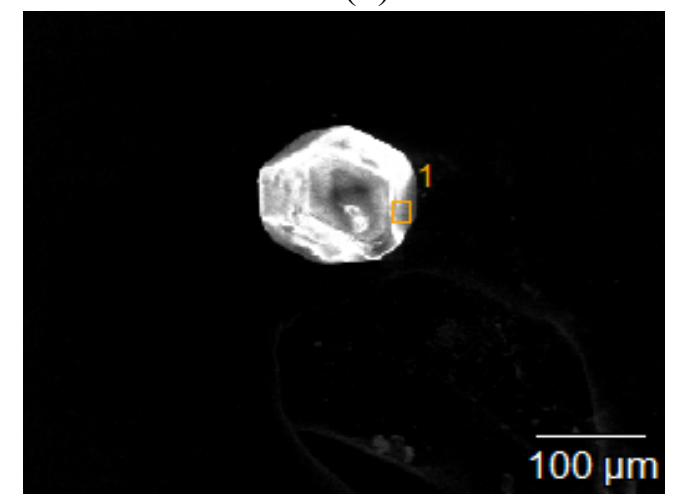

(d)

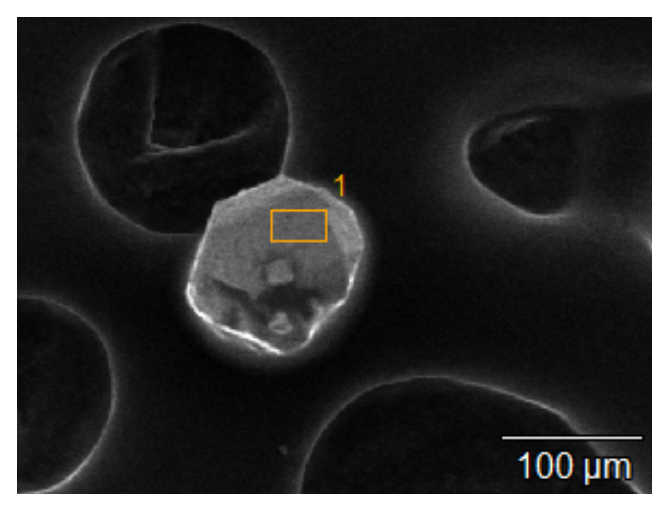

(f) 


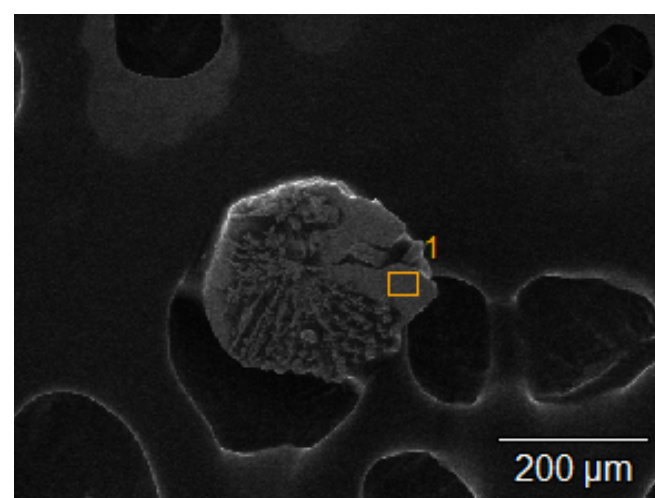

$(\mathrm{g})$

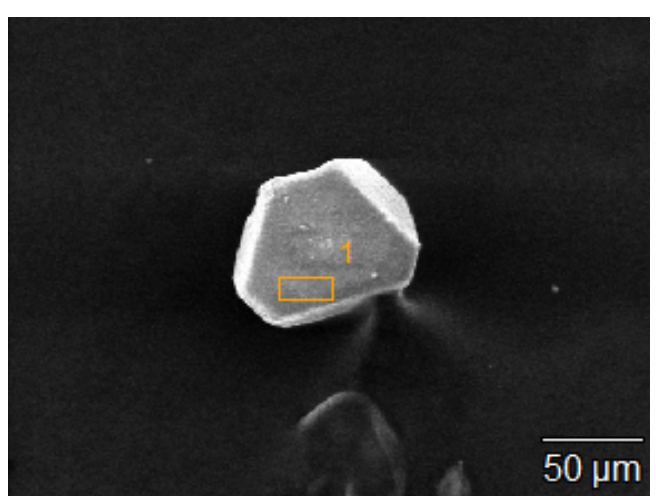

(h)

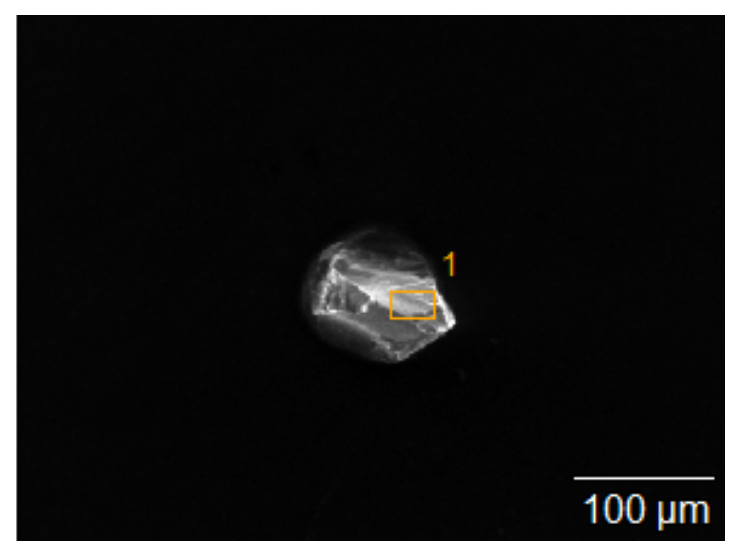

(i)

Figure S2. SEM images of crystals of $\mathrm{Cs}_{5} \mathrm{Y}_{2} \mathrm{Cl}_{11}\left(\mathrm{H}_{2} \mathrm{O}\right)_{17}$ (a), $\mathrm{Cs}_{5} \mathrm{Lu}_{2} \mathrm{Cl}_{11}\left(\mathrm{H}_{2} \mathrm{O}\right)_{17}$ (b), $\mathrm{Cs}_{2} \mathrm{EuCl}_{5}\left(\mathrm{H}_{2} \mathrm{O}\right)_{10}$ (c), $\mathrm{Cs}_{7} \mathrm{GdCl}_{10}\left(\mathrm{H}_{2} \mathrm{O}\right)_{8}(\mathrm{~d}), \mathrm{Cs}_{10} \mathrm{~Tb}_{2} \mathrm{Cl}_{17}\left(\mathrm{H}_{2} \mathrm{O}\right)_{14}\left(\mathrm{H}_{3} \mathrm{O}\right)$ (e), $\mathrm{Cs}_{2} \mathrm{DyCl}_{5}\left(\mathrm{H}_{2} \mathrm{O}\right)_{6}$ (f), $\mathrm{Cs}_{7} \mathrm{HoCl}_{10}\left(\mathrm{H}_{2} \mathrm{O}\right)_{8}(\mathrm{~g}), \mathrm{Cs}_{8} \mathrm{Er}_{3} \mathrm{Cl}_{17}\left(\mathrm{H}_{2} \mathrm{O}\right)_{25}(\mathrm{~h})$, and $\mathrm{Cs}_{2} \mathrm{YbCl}_{5}\left(\mathrm{H}_{2} \mathrm{O}\right)_{6}(\mathrm{i})$. 
Table S1. Elemental composition determined by EDS

\begin{tabular}{|c|c|c|c|c|c|c|c|}
\hline \multicolumn{2}{|c|}{$\mathrm{Cs}_{5} \mathrm{Y}_{2} \mathrm{Cl}_{11}\left(\mathrm{H}_{2} \mathrm{O}\right)_{17}$} & \multicolumn{2}{|c|}{$\mathrm{Cs}_{5} \mathrm{Lu}_{2} \mathrm{Cl}_{11}\left(\mathrm{H}_{2} \mathrm{O}\right)_{17}$} & \multicolumn{2}{|c|}{$\mathrm{Cs}_{2} \mathrm{EuCl}_{5}\left(\mathrm{H}_{2} \mathrm{O}\right)_{10}$} & \multicolumn{2}{|c|}{$\mathrm{Cs}_{7} \mathrm{GdCl}_{10}\left(\mathrm{H}_{2} \mathrm{O}\right)_{8}$} \\
\hline Element & Atom $\%$ & Element & Atom \% & Element & Atom $\%$ & Element & Atom $\%$ \\
\hline Cs & 17.50 & Cs & 17.75 & Cs & 21.83 & Cs & 31.23 \\
\hline $\bar{Y}$ & 7.71 & $\mathrm{Lu}$ & 8.01 & $\mathrm{Eu}$ & 4.57 & $\mathrm{Gd}$ & 5.93 \\
\hline $\mathrm{O}$ & 31.51 & $\mathrm{O}$ & 28.64 & $\mathrm{O}$ & 32.72 & $\mathrm{O}$ & 19.82 \\
\hline $\mathrm{Cl}$ & 43.28 & $\mathrm{Cl}$ & 45.6 & $\mathrm{Cl}$ & 40.87 & $\mathrm{Cl}$ & 43.02 \\
\hline \multicolumn{2}{|c|}{$\mathrm{Cs}_{10} \mathrm{~Tb}_{2} \mathrm{Cl}_{17}\left(\mathrm{H}_{2} \mathrm{O}\right)_{14}\left(\mathrm{H}_{3} \mathrm{O}\right)$} & \multicolumn{2}{|c|}{$\mathrm{Cs}_{2} \mathrm{DyCl}_{5}\left(\mathrm{H}_{2} \mathrm{O}\right)_{6}$} & \multicolumn{2}{|c|}{$\mathrm{Cs}_{7} \mathrm{HoCl}_{10}\left(\mathrm{H}_{2} \mathrm{O}\right)_{8}$} & \multicolumn{2}{|c|}{$\mathrm{Css}_{2} \mathrm{Er}_{3} \mathrm{Cl}_{17}\left(\mathrm{H}_{2} \mathrm{O}\right)_{25}$} \\
\hline Element & Atom $\%$ & Element & Atom $\%$ & Element & Atom $\%$ & Element & Atom $\%$ \\
\hline $\mathrm{Cs}$ & 21.01 & $\mathrm{Cs}$ & 19.87 & $\mathrm{Cs}$ & 17.84 & $\mathrm{Cs}$ & 18.94 \\
\hline $\mathrm{Tb}$ & 10.52 & Dy & 11.04 & Ho & 8.82 & $\mathrm{Er}$ & 10.99 \\
\hline $\mathrm{O}$ & 11.95 & $\mathrm{O}$ & 18.25 & $\mathrm{O}$ & 21.14 & $\mathrm{O}$ & 21.93 \\
\hline $\mathrm{Cl}$ & 56.52 & $\mathrm{Cl}$ & 50.84 & $\mathrm{Cl}$ & 52.19 & $\mathrm{Cl}$ & 48.14 \\
\hline
\end{tabular}

\begin{tabular}{|c|c|}
\hline \multicolumn{2}{|c|}{$\mathbf{C s}_{\mathbf{2}} \mathbf{Y b C l}_{\mathbf{5}}\left(\mathbf{H}_{\mathbf{2}} \mathbf{O}\right)_{\mathbf{6}}$} \\
\hline Element & Atom \% \\
\hline $\mathrm{Cs}$ & 18.85 \\
\hline $\mathrm{Yb}$ & 9.08 \\
\hline $\mathrm{O}$ & 24.76 \\
\hline $\mathrm{Cl}$ & 47.31 \\
\hline
\end{tabular}




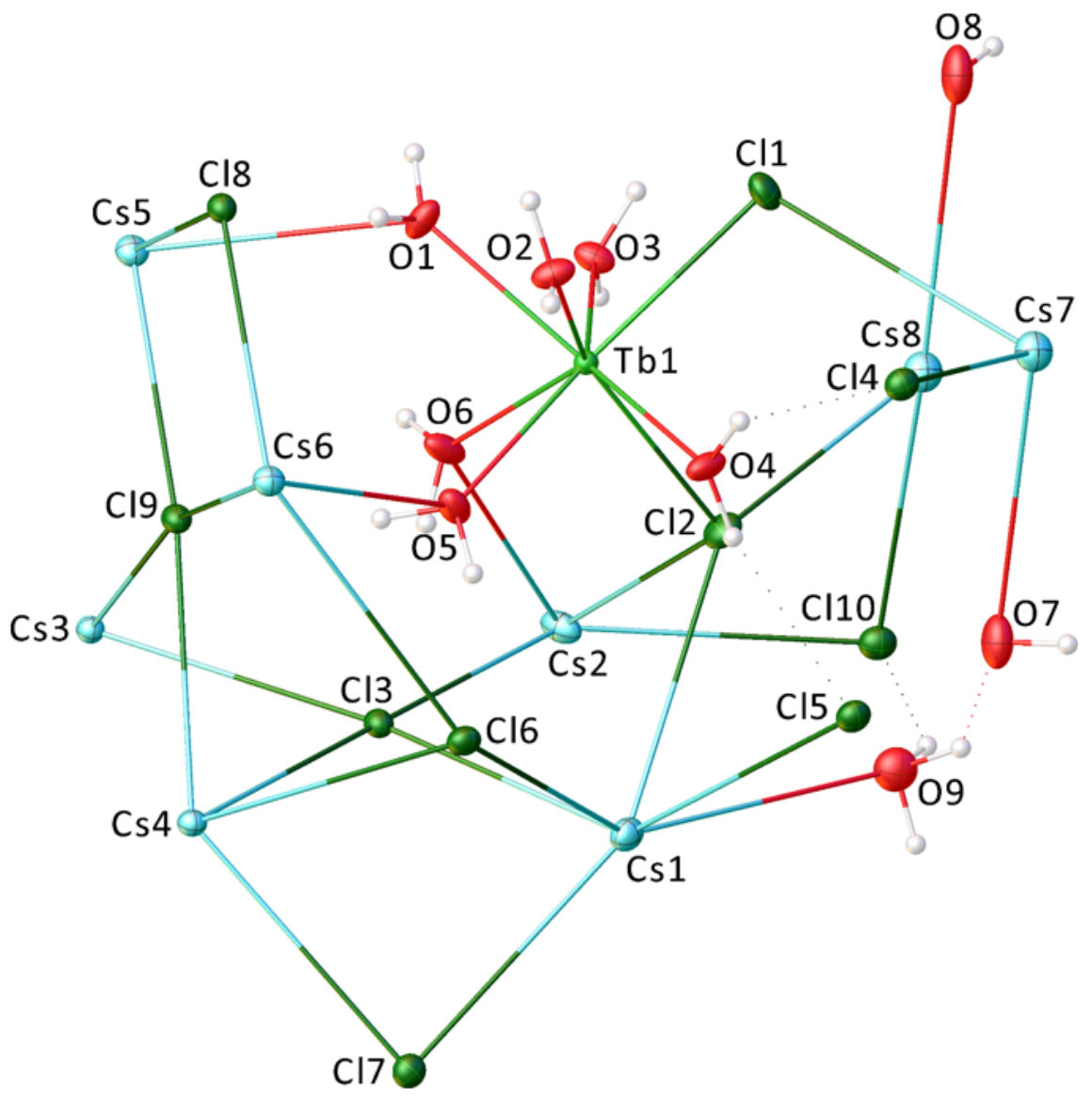

Figure S3. Asymmetric unit of $\mathrm{Cs}_{10} \mathrm{~Tb}_{2} \mathrm{Cl}_{17}\left(\mathrm{H}_{2} \mathrm{O}\right)_{14}\left(\mathrm{H}_{3} \mathrm{O}\right)$. Displacement ellipsoids drawn at the $50 \%$ probability level. $\mathrm{O} 9$ is a disordered hydronium ion. $\mathrm{O} 8$ and $\mathrm{O} 9$ are water molecules located on mirror planes. Cs3-Cs8, Cl8-Cl10 and O7-O9 are located on or are disordered across (O9) mirror planes. Tb1, Cs1-Cs2, $\mathrm{Cl1}-\mathrm{Cl}$ and $\mathrm{O} 1-\mathrm{O} 6$ are located on general positions. 


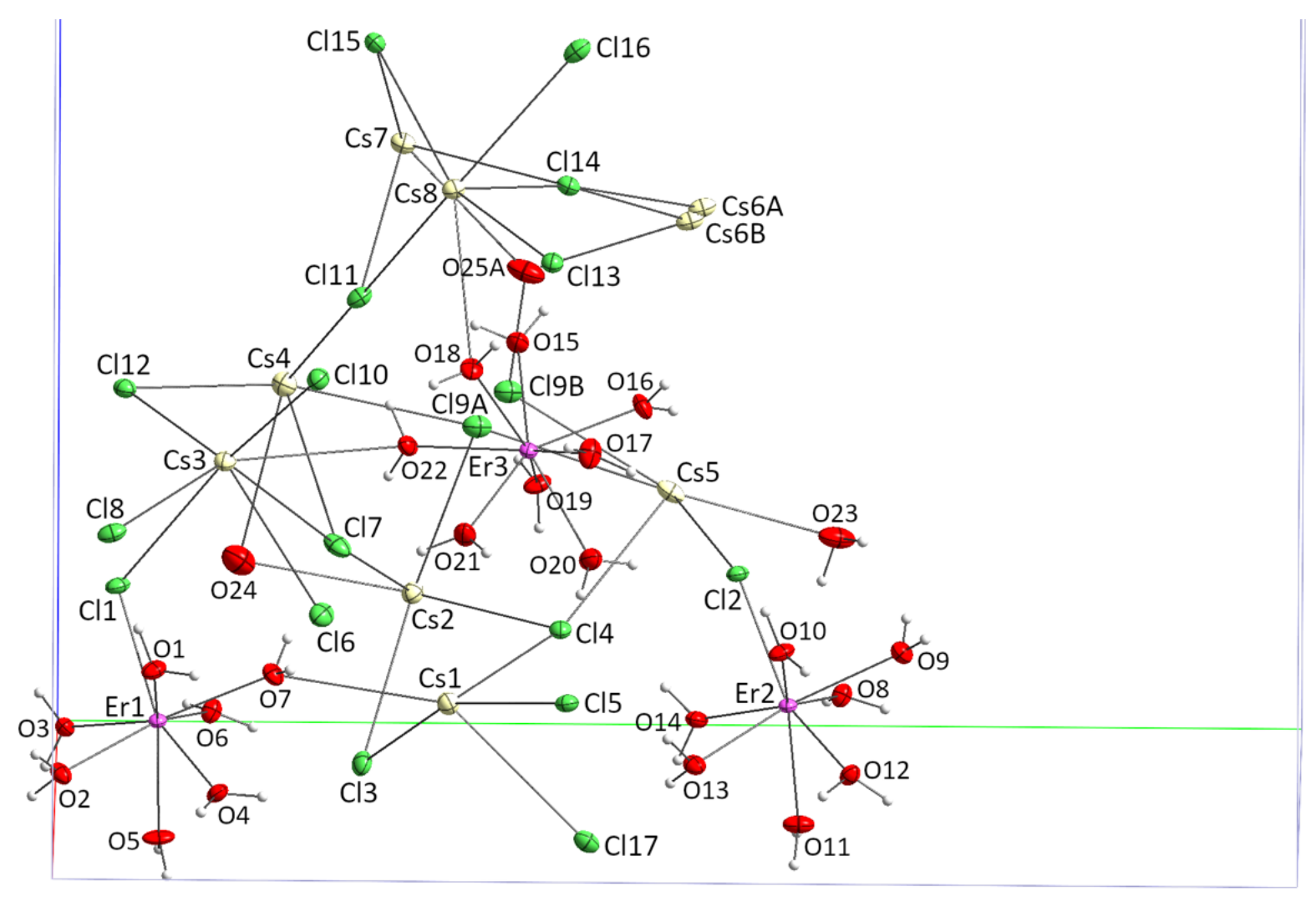

Figure S4. Asymmetric unit for $\mathrm{Cs}_{8} \mathrm{Er}_{3} \mathrm{Cl}_{17}\left(\mathrm{H}_{2} \mathrm{O}\right)_{25}$ with $50 \%$ probability displacement ellipsoids. 


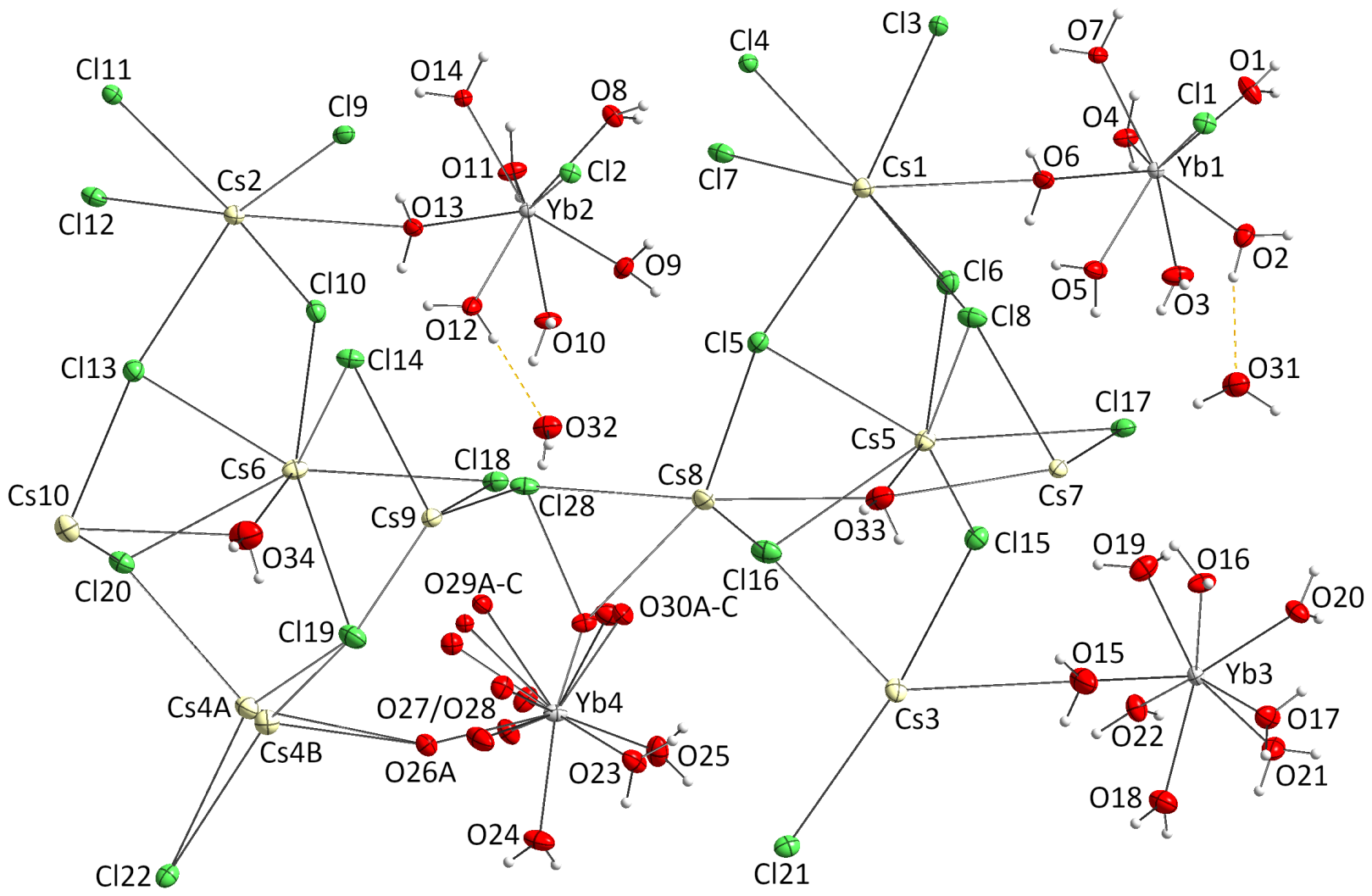

Figure S5. Asymmetric unit for $\mathrm{Cs}_{5} \mathrm{Yb}_{2} \mathrm{Cl}_{11}\left(\mathrm{H}_{2} \mathrm{O}\right)_{17}$ with displacement ellipsoids drawn at the 50\% probability level. 